\title{
Application of Histone Deacetylase Inhibitors in Renal Interstitial Fibrosis
}

\author{
Ling Nie Yong Liu Bo Zhang Jinghong Zhao \\ Department of Nephrology, Institute of Nephrology of Chongqing and Kidney Center of PLA, Xinqiao Hospital, \\ Army Medical University (The Third Military Medical University), Chongqing, China
}

\section{Keywords}

Renal interstitial fibrosis · Histone deacetylases · Signaling pathway $\cdot$ Inhibitor

\begin{abstract}
Background: Renal interstitial fibrosis is characterized by the accumulation of extracellular matrix proteins, which is a common feature of chronic kidney diseases. Summary: Increasing evidence has shown the aberrant expression of histone deacetylases (HDACs) in the development and progression of renal fibrosis, suggesting the possibility of utilizing HDAC inhibitor (HDACi) as therapeutics for renal fibrosis. Recent studies have successfully demonstrated the antifibrotic effects of HDACis in various animal models, which are associated with multiple signaling pathways including TGF- $\beta$ signaling, EGRF signaling, signal transducer and activator of transcription 3 pathway, and JNK/Notch2 signaling. This review will focus on the utilization of HDACi as antifibrotic agents and its relative molecular mechanisms. Key Messages: HDACis have shown promising results in antifibrotic therapy, and it is rational to anticipate that HDACis will improve clinical outcomes of renal fibrosis in the future.

(c) 2020 The Author(s)

Published by S. Karger AG, Basel
\end{abstract}

\section{Introduction}

Renal interstitial fibrosis, characterized by the activation and proliferation of fibroblasts, and subsequent production of excessive amounts of extracellular matrix (ECM) deposition in response to acute and chronic damage, is a common feature of chronic kidney diseases (CKD) and contributes to the deterioration of renal function [13]. Many cytokines participate in this process, leading to differentiation of renal interstitial fibroblasts into the activated myofibroblast phenotype, which can be identified by markable expression of $\alpha$-smooth muscle actin ( $\alpha$-SMA) [4-7]. Of those cytokines, transforming growth factor- $\beta 1$ (TGF- $\beta 1$ ) is considered as the master regulator in renal fibrosis [8]. TGF- $\beta 1$ is mainly secreted by macrophages responding to inflammation in injured tissues, and its interaction with receptor leads to activation of signal transducer and activator of transcription 3 (STAT3), EGRF and JNK/Notch2 signaling pathways, which promote the excessive deposition of ECM components such as collagen [9]. Other types of cells including tubular epithelial cells, hematopoietic cells, and endothelial cells are also involved in the production of collagen [10]. Antifibrotic drugs focus on gene expression regulation have been developed, but the clinic outcomes were unsatisfactory.

\begin{tabular}{ll}
\hline karger@karger.com & (c) 2020 The Author(s) \\
Published by S. Karger AG, Basel Oparger & Koen ciccess \\
www.karger.com/kdd & This article is licensed under the Creative Commons Attribution- \\
Karger & NonCommercial-NoDerivatives 4.0 International License (CC BY- \\
NC-ND) (http://www.karger.com/Services/OpenAccessLicense). \\
Usage and distribution for commercial purposes as well as any dis- \\
tribution of modified material requires written permission.
\end{tabular}

Jinghong Zhao, MD, PhD

Department of Nephrology, Xinqiao Hospital

Army Medical University (The Third Military Medical University)

Xinqiao Street 83, Shapingba, Chongqing 400037 (China)

zhaojh@tmmu.edu.cn 
Increasing evidence elucidates that epigenetic mechanisms, such as histone acetylatic modifications, play an important role in interstitial fibrosis process [11, 12]. Acetylation of core histones is governed by opposing actions of a variety of histoneacetyltransferases (HATs) and histone deacetylases (HDACs) [13]. HATs catalyze the transfer of acetyl groups from acetyl-CoA to the lysine $\varepsilon$-amino groups on the $\mathrm{N}$-terminal tails of histones, which weakens the interaction of the histone tail and DNA, promoting transcriptional activation. In contrast, HDACs remove the acetyl groups from acetylated histones, increase chromatin condensation, and suppress gene transcription [14]. Aberrant expressions of HDACs and subsequent epigenetic modifications have been extensively observed in various animal models of interstitial fibrosis, and inhibitory effects against tissue fibrosis by HDACs inhibitors have also been demonstrated in multiple organs, such as heart $[15,16]$, liver $[17,18]$, and kidney $[19,20]$, which presents the possibility of using HDACs as targets in the treatment of chronic fibrotic diseases. In this minireview, we will discuss HDACs inhibitors' application and molecular mechanism in renal interstitial fibrosis.

\section{HDACs in Mammalian Cell}

The biochemical function of HATs is to catalyze the acetylation of histones, which results in an open structure of the DNA. Hyperacetylation of core histone facilitates the binding of transcription factors and promotes gene expression. On the contrary, HDACs remove acetylgroups from hyperacetylated histones and counteract with HATs. HATs and HDACs are recruited to gene promoters by DNA-binding proteins that recognize given DNA sequences and provide specific modulation on gene expression.

There are 18 characterized members of HDACs in human, which can be grouped into 4 classes based on biological function and DNA sequence similarity [21]. The class I and II HDACs are considered as the "classical" HDACs, whose activities are inhibited by small chemicals such as trichostatin A (TSA). Class III HDACs represent the silent information regulator 2 family of nicotinamide adenine dinucleotide-dependent HDACs (SIRT1-7), which share structural and functional similarities with the yeast silent information regulator 2 protein [22]. Finally, class IV HDAC is the newly discovered HDAC11. HDAC11 is most closely related to class I HDACs, but since the overall sequence similarities are very low, it can- not be grouped into any of the other existing classes. In mammalian cells, different tissue distribution and its cellular location of each member is summarized in Table 1.

At present, the expression profiles and distribution of HDACs in the kidney have not been completely clarified. By using specific inhibitors, HDACs have been shown to be involved in a variety of important cellular processes such as cell proliferation, survival, differentiation, and cell apoptosis. Class I HDACs are expressed ubiquitously, localized to the nucleus, and interact with co-repressor complexes to exert their function. In the kidney, its expression was found in renal fibroblasts and renal tubular cells [23]. Class II HDACs are thought to have tissue-specific roles and are expressed in the renal tubules [24]. Class III HDACs are members of the sirtuin family and are characterized by a sirtuin core domain. Cellular distribution of this Class HDACs includes nucleus (SIRT1, SIRT6, and SIRT7), mitochondria (SIRT3, SIRT4, and SIRT5), and cytoplasm (SIRT2). SIRT1 and SIRT2 are found expressed in renal fibroblasts and tubular cell, while cellular distribution of other members in this subfamily is not clear. Studies have demonstrated the role of class III HDACs in tissue fibrosis, but results regarding SIRT1 in renal fibrosis are controversial, as both activation and inhibition of SIRT1 can attenuate renal fibrosis [25-29]. HDAC 11 is the sole member of class IV and is localized to the nucleus and may be involved in regulating the expression of interleukin 10. Expression of HDAC11 was found in renal tubules, where it negatively regulated PAI-1 expression and might be a novel target in ischemia and reperfusion injury [30]. However, the relationship between HDAC11 and renal fibrosis is unclear.

\section{Aberrant Expressions of HDACs in Renal Interstitial Fibrosis}

The molecular process of renal interstitial fibrosis is accompanied by aberrant gene expression that confers proliferation and activation of interstitial cells. As the fundamental biochemical function of HDACs is to remove acetyl group from hyperacetylated histones, the gene expression of HDACs is thought to be associated with renal interstitial fibrosis. Moreover, the aberrant gene expression of HDACs in interstitial cells can provide an interpretation of deregulated gene expression of interstitial cells.

Many studies indicate that aberrant expression of HDACs was found in various models of renal interstitial fibrosis. The most popular model of renal interstitial fi- 
Table 1. Classification of HDACs and its expression in kidney

\begin{tabular}{llllll}
\hline Group & Member & Cellular distribution & Renal location & Aberration & Reference \\
\hline Class I & HDAC1 & Nucleus & Renal fibroblasts & Up & {$[36-39]$} \\
& HDAC2 & & and tubular cell & Up & {$[36-39]$} \\
& HDAC3 & & Down/up & {$[37,40]$} \\
& HDAC8 & & Down/up & {$[38,43]$} \\
\hline Class II & HDAC4 & Nucleus/cytoplasm & Renal tubules & Up & {$[39]$} \\
& HDAC5 & & & Up & {$[38]$} \\
& HDAC6 & & Up & {$[38,41]$} \\
& HDAC7 & & Up & {$[36]$} \\
& HDAC9 & & ND & {$[38]$} \\
\hline Class III & HDAC10 & & Up & {$[23]$} \\
& Sirt1 & Cytoplasm/nucleus/ & Renal tubules and & Down & {$[25]$} \\
& Sirt3 & mitochondria & fibroblasts & Up & {$[27]$} \\
& Sirt4 & & & Down & ND \\
\hline Class IV & Sirt5 & & & ND & ND \\
\hline
\end{tabular}

HDACs, histone deacetylases.

brosis is unilateral ureteral obstruction (UUO) and diabetic nephropathy $[31,32]$. Relative fewer studies focus on drug-induced or IRI-induced renal damage-associated fibrosis [33, 34]. In UUO mice, increased gene expression of HDAC-1, -2 , and -7 was found both at transcriptional and translational levels [35]. Increase of HDAC-1 and -2 was also confirmed by other studies $[36,37]$. In a mouse model of diabetic kidneys diseases (DKD), the activity of HDAC-1, -2 , and -4 was found to be elevated in STZ-inducted renal cortex, while the activity of HDAC-3, -5 , and -8 was not significantly changed [38]. In the kidneys of adenine-fed CKD mice, HDAC 3 was found to be upregulated, and selective HDAC3 inhibition effectively alleviated kidney injury [39]. In angiotensin II-infused mice, hypertensive stimuli enhanced the expression of HDAC6, and the inhibition of HDAC6 prevented fibrosis and inflammation [40]. Similarly, downregulation of SIRT3 was found in angiotensin II-infused mice, while overexpression of SIRT3 repressed the excessive production of mitochondrial superoxide [41]. All these studies show that members of HDACs are aberrantly expressed in different animal models, although not all the members of HDACs are fully investigated (Table 1).

The changes in HDAC expression are thought to be involved in transmission of signals under the condition of renal interstitial fibrosis. The induction of HDACs likely contributes to the reduced level of histone acetylation and altered gene expression in the injured kidney. This forms the molecular basis to treat renal interstitial fibrosis with HDAC inhibitors (HDACis).

\section{Classification of HDACi}

The development of HDACi has a long history, and a number of structurally diverse HDACis have been identified. Many HDACi are currently being evaluated in clinical trials, especially for cancer therapy [42]. In 2003, Mishra et al. [43] found that TSA treatment could significantly decrease the urine-protein excretion and the proliferative hallmarks of glomerulonephritis associated with systemic lupus erythematosus-induced lupus in mice for the first time. Thereafter, various HDACis have been investigated for their antifibrotic and anti-inflammatory effects in renal disease. The body of HDACis still gets growing, with many new chemicals derived from natural product are found to be potential HDACi. Based on their chemical structure, HDACi can be classified into the following categories [44]: hydroxamic acids (such as TSA and suberoylanilide hydroxamic acid); cy- 
Table 2. HDACi applied in animal models of renal fibrosis

\begin{tabular}{llll}
\hline HDACi & Specificity & Animal model & Reference \\
\hline TSA & Class I/II & STZ-induced diabetic kidney & {$[39]$} \\
& & UUO model & {$[36,39,46,55,68]$} \\
& & Doxorubicin-induced nephropathy & {$[35]$} \\
& & IRI-induced fibrosis & {$[34]$} \\
\hline VPA & Adenine CKD model & {$[35]$} \\
\hline MS-275 & & UUO model & {$[38]$} \\
& & Doxorubicin-induced nephropathy & {$[38]$} \\
\hline NaB & Class I & STZ-induced diabetic kidneys & {$[39,48]$} \\
\hline SAHA & & UUO model & {$[37]$} \\
\hline SB939 & Pan-HDACi & STZ-induced diabetic kidney & {$[34]$} \\
\hline Tubastatin A & Class I/II & STZ-induced diabetic kidney & {$[33,66]$} \\
\hline
\end{tabular}

HDACs, histone deacetylases; TSA, trichostatin A; VPA, valproic acid; SAHA, suberoylanilide hydroxamic acid; HDACi, HDAC inhibitor; UUO, unilateral ureteral obstruction.

clic peptide (such as FK228); short-chain and aromatic fatty acids (such as butyrate); benzamides (such as MS275), and miscellaneous compounds (such as depudecin). Many inhibitors are HDAC type specific, while some inhibitors are member specific. For example, valproic acid (VPA) exerts inhibition of both class I and II HDACs, with a high potency for class I HDACs, while Tubastatin A is a specific selective inhibitor of HDAC6. Still, some inhibitors can inhibit all HDAC isoforms nonspecifically (so-called pan-inhibitors). For example, SB939 (pracinostat) is a new orally active hydroxamatebased HDACi currently in phase II clinical trials, which can potently inhibit class I, II, and IV HDACs with excellent pharmacokinetic properties [45]. The often-used HDACi in renal interstitial fibrosis and their characters are listed in Table 2.

\section{Cellular and Molecular Mechanisms for HDACi in Kidney Fibrosis}

As renal fibrosis is one of the typical complex diseases, many genes are involved in the fibrogenesis. Various molecular mechanisms are proposed to explain the renal interstitial fibrosis. Accordingly, the mechanisms of HDACi vary in different animal models (Fig. 1).

\section{Cellular Mechanisms of HDACi}

\section{Inhibition of Fibroblast Activation and Proliferation}

Regardless of the initiating events, a feature common to renal fibrosis is the activation of ECM-producing myofibroblasts, which are derived from active fibroblast-type cells. $\alpha$-SMA is a representative marker of myofibroblast activation. In the kidney, the most abundant fibroblasttype cells are mesangial cells and fibroblasts. Another source of fibroblast-type cells is epithelial-mesenchymal transition (EMT) of tubular epithelial cells (also see below).

In animal models of renal fibrosis, fibroblast activation and proliferation can be represented by gene expression of a-SMA, fibronectin, or collagens. These proteins are also taken as molecular markers of renal fibrosis. In other studies, connective tissue growth factor and PAI-1 are also taken as fibrosis markers. Connective tissue growth factor is a matricellular protein related to tissue and wound repair and fibrotic pathology, while PAI-1 protein can activate protease inhibitors to inhibit ECM degradation [46]. In all the animal studies, the tested HDACi can reverse the gene expression of fibrosis markers, indicating the inhibition of fibroblast activation and proliferation. Moreover, the reversed gene expression of fibrosis markers can be exerted by the direct regulation of histone acetylation of promoter regions in these genes [47]. In 


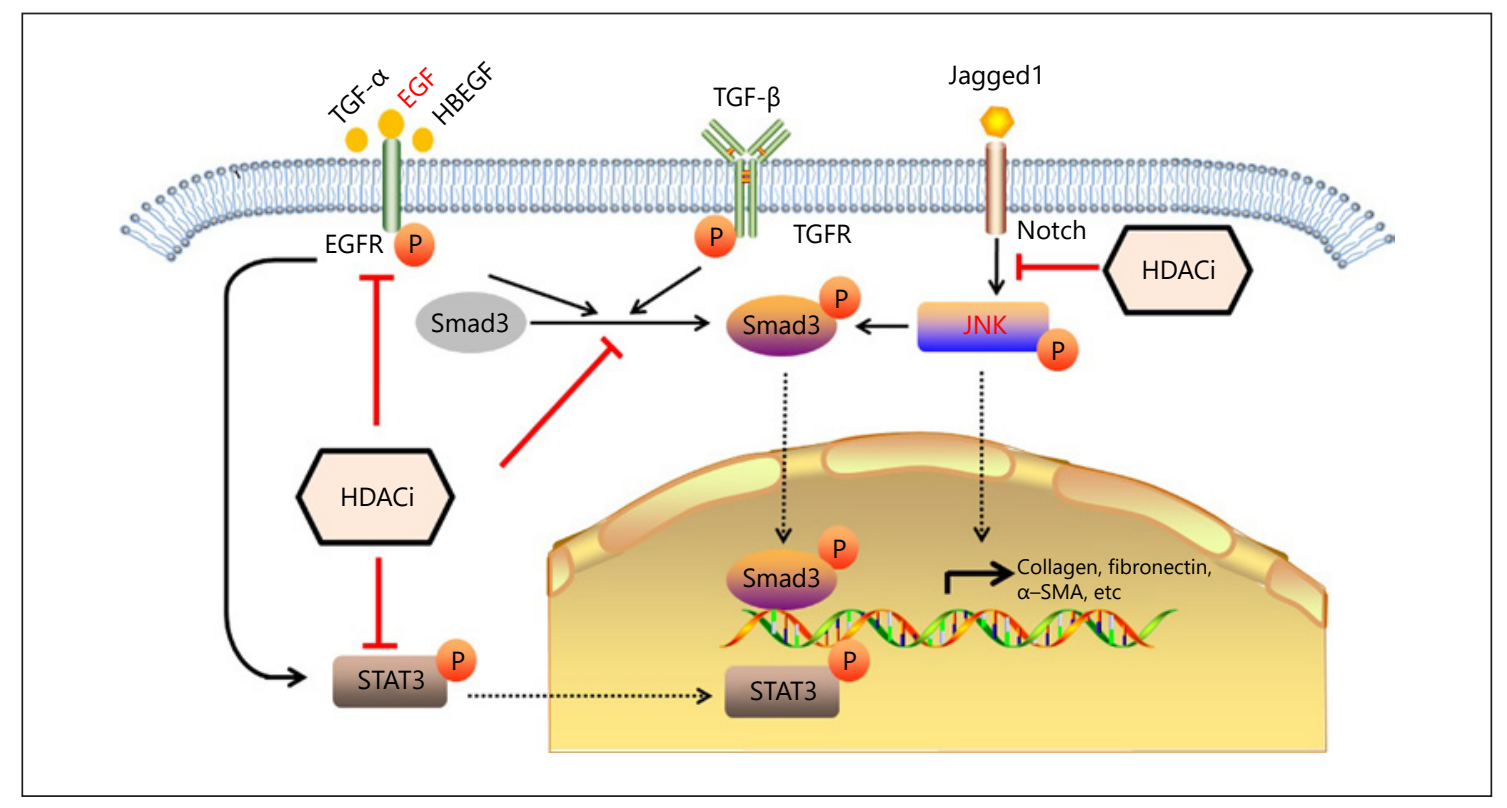

Fig. 1. Effects of HDACi and its associated signaling pathways in renal interstitial fibrosis. HDACi is showing to protect against interstitial fibrosis through multiple signaling pathway as indicated. EGF, epidermal growth factor; TGF, transforming growth factor; TGFR, TGF-receptor; HDACi, histone deacetylases inhibitor; STAT3, signal transducer and activator of transcription 3; $\alpha$-SMA, $\alpha$-smooth muscle actin.

vitro studies also showed that the tested HDACi could inhibit fibroblast proliferation directly, as detected by MTT assay or counting cells $[36,48]$. Thus, these studies clearly show HDACi can inhibit fibroblast activation and proliferation.

\section{Inhibition of Circulating Fibrocytes}

As mentioned above, besides activation of ECM-producing myofibroblasts, another main source of ECMproducing cells are collagen-producing hematopoietic cells, which are also called fibrocytes and thought to arise from circulating monocytes in a process called monocyte/ macrophage-to-mesenchymal transition [10]. By using of lineage tracing and collagen I reporter mice, recent researches revealed that the number of collagen-expressing cells may not correlate with their actual production of collagen, because some of these cells may only produce very low amounts of collagen or contribute to fibrosis only indirectly [49]. While fibrocytes were reported to play an important role in the development of renal fibrosis in UUO and adenine-induced nephropathy models, by which up to $50 \%$ of the deposited collagen I was derived from hematopoietic cells. Thus, inhibition of the differentiation of fibrocyte precursors into mature, collagen-producing fibrocytes may prevent renal fibrosis.
Class I HDAC-selective inhibitor MGCD0103 was reported to block angiotensin II (Ang II)-mediated increases in fibrocytes in the heart completely and also decrease circulating levels of fibrocytes, although it was not able to block the recruitment of fibrocytes to the heart. MGCD0103 blocked fibrocytes differentiation as effectively as serum amyloid $\mathrm{P}$ by suppressing ERK1/2 phosphorylation in fibrocytes precursors [50]. More studies needed to prove inhibition of HDACi on circulating fibrocytes in renal fibrosis models.

\section{Inhibition of Tubular Cell Death and EMT}

Many factors involved in the causation of renal fibrosis have direct effects on renal tubules, especially tubular epithelial cells. Loss of tubular cells contributes to deterioration of renal function, which is the case under the condition of acute kidney injury. Inhibition of tubular cell death was observed in UUO mice treated with TSA [51]. Moreover, some profibrogenic factors can induce phenotypic transition of renal tubules, which is termed as EMT. The pilot study was performed in a mouse tissue, in which the presence of EMT in renal fibrosis was first demonstrated by the using a fibroblast-specific protein marker [52]. Then the existence of EMT in remnant kidney after $5 / 6$ nephrectomy and in UUO-induced kidney was also 
confirmed by different groups $[53,54]$. Thus, it is widely accepted that fibroblast-type cells derived from tubular epithelial cells play an important role in renal fibrosis.

The process of tubular EMT is defined as loss of the tubular epithelial phenotype and gain of mesenchymal features. At molecular level, EMT is characterized by a decrease in the expressions of E-cadherin and zonula occludens protein with an acquisition of de novo a-SMA expression, as well as vimentin and fibronectin [55]. Moreover, morphologic and phenotypic evidence has also been noticed during the process of EMT. Active participation of EMT in CKD and acute kidney injury has also been reported in animal models and cell models. In our previous study, we found that treatment with high uric acid induced EMT of human tubular epithelial cells [56].

EMT under the condition of renal fibrosis can be reversed by HDACi treatment. In a cell model of HK-2 cells treated with TGF- $\beta$, various HDACi including TSA, MS275, PCI34051, and LMK235 were applied to inhibit ECM proteins expression, while TGF- $\beta 1$-induced E-cadherin downregulation was only observed in HK-2 cells treated with TSA or MS275 [57]. This work was coincident with a previous report in which TSA was applied to inhibit EMT induced by TGF- $\beta$ in HK- 2 cells, and the acetylation level of EMT marker was significantly increased by TSA treatment [58]. Similarly, the inhibition of EMT induced by TGF- $\beta$ was also observed in SB939treated HK-2 cells, which demonstrated the in vitro function of HDACi [59]. In STZ-induced diabetic kidneys, the nonselective HDACi TSA could efficiently inhibit EMT in vivo, as determined by Western blotting [38]. In the same animal model, the antifibrotic and renoprotective effects of VPA and $\mathrm{NaB}$ via inhibition of EMT were also detected [60, 61]. In UUO-induced renal fibrosis, the existence of EMT was demonstrated by the abundant cells coexpressing both $\alpha$-SMA and tubular marker, which indicated that these cells were at transitional stage between epithelia and mesenchyme [62]. However, the protein expression of EMT marker E-cadherin was controversial [37]. As EMT can happen in UUO-induced kidney, it can be speculated these HDACi have the ability to inhibit EMT in vivo, although the data in UUO model are not available at present. Briefly, tubular EMT plays an important role in the pathogenesis of renal interstitial fibrosis, inhibition of EMT by HDACi will undoubtly alleviate renal fibrosis both in vitro and in vivo.
Inhibition of Renal Inflammation and Immune Cells

The relationship between interstitial inflammation and renal fibrosis is far from clear. Renal fibrosis is characterized by sustained inflammation, including inflammatory cell infiltration and secretion of cytokines. Besides immune factors, a wide range of nonimmune factors, including reactive oxygen species and advanced glycation end products, have been implicated in driving renal fibrosis via inflammation. Under the condition of renal damage, inflammation is precisely regulated by the immune system for tissue repairing, which seeks to eliminate the cause of injury. However, the sustained inflammatory response also can stimulate an inappropriate profibrotic response. Therefore, regulation of inflammatory response is a potential target for antifibrotic therapy.

Owing to the progresses in the past years, it is now clear that many elements of the innate and adaptive immune response participate in renal fibrosis. Once renal damages happen, acute inflammation and activation of innate immune mediators will be triggered. Subsequently, cytokines from renal intrinsic cells and innate immune cells will influence the activation of the adaptive immune response. Also, renal damages can directly activate the adaptive immune response. Inflammatory and immune mediators attempt to eliminate the inciting factors while activating the quiescent fibroblasts into myofibroblasts. Failure to eliminate the inciting factors can exacerbate the inflammatory response, which ultimately results in renal fibrosis.

In the classic UUO model of renal fibrosis, renal tubular cells can express several chemokines that induce the migration of inflammatory cells into the interstitium. This was observed in many studies. Although the role of macrophages in acute and chronic kidney injury is complex, For example, inflammatory M1 macrophages and anti-inflammatory M2 macrophages [63], the inhibition of initial macrophage influx by TSA treatment has been shown to attenuate tubulointerstitial injury in UUO model [35]. Many other HDACi also have the ability to inhibit macrophage infiltration. FR276457 is a hydroxamic acid derivative that inhibits both class I and II HDACs. Treatment with FR276457 after UUO resulted in decreased renal MCP-1 levels, a chemotactic cytokine that attracts macrophages to the kidney shortly after UUO [64]. In Ang II-induced hypertension mice, the HDAC6selective inhibitor Tubastatin A reduced renal inflammation, as determined by qRT-PCR of MCP- 1 and TNF- $\alpha$ [37]. Similarly, increased expression of inflammatory cytokines (TNF- $\alpha$ and IL-6) and profibrotic cytokines (TGF- $\beta$ and IL-13) in obstructed kidneys were signifi- 
cantly reduced by SB939, a new orally active hydroxamate-based HDACi currently in phase II clinical trials [65]. Beside macrophages, other immune cells, which participate in renal fibrosis, can also be regulated by HDACi treatment. Like macrophages, T-cell infiltration is evident in most types of human and experimental CKD models [66]. In addition to produce chemokines and cytokines, $\mathrm{T}$ cells can participate in renal fibrosis by regulating the differentiation of fibrocytes. Moreover, T cells have also been proposed to promote renal fibrosis via EMT through the production of TGF- $\beta 1$.

Recently, the function of FOXP ${ }^{3+} \mathrm{IL}-17^{+} \mathrm{T}$ cells in UUO model was revealed by $\mathrm{Wu}$ et al. [67], whose results showed that the attenuation of renal fibrosis by TSA was associated with the plasticity of FOXP ${ }^{3+} \mathrm{IL}-17^{+} \mathrm{T}$ cells. This is a new mechanism to explain the biological effects of HDACi. On the other hand, the chemokines and cytokines produced by renal intrinsic cells contribute to renal fibrosis. It is hard to discriminate the cell source of the chemokines and cytokines from renal samples directly. However, the in vitro model can answer the question. Treatment with TNF- $\alpha$ in rat tubular epithelial cells (NRK 52E) led to upregulation of MCP-1 and CSF-1, a chemokine involved in the initiation of tubulointerstitial injury, while this upregulation was significantly reversed by TSA [35]. This in vitro data suggested that HDACi could inhibit the production of cytokine and chemokines in activated tubular cells. Recently, anti-inflammatory effect of TSA was demonstrated in a model of cisplatin nephrotoxicity, in which TSA upregulated a novel anti-inflammatory protein, and activated microglia/macrophage WAP domain protein in epithelial cells [68]. Thus, the gene expression of inflammatory cytokines and chemokines in renal intrinsic cells can be efficiently inhibited by treatment of HDACi, which can in turn ameliorate renal fibrosis.

\section{Molecular Mechanisms of HDACi}

\section{Inhibition of TGF- $\beta$ Signaling}

TGF- $\beta$ is a master regulator of renal fibrosis, as well as fibrosis in other organs [69]. Numerous studies have manifested that increased expression and activation of profibrotic TGF- $\beta$ is associated with renal interstitial fibrosis. TGF- $\beta$ is also taken as the predominant pathogenic factor that drives glomerular and interstitial fibrosis. The canonical TGF- $\beta$ signaling pathway involves the phosphorylation and activation of Smad 2 and 3 through TGF- $\beta$ receptor 1 . Subsequently, the Smad complex translocates to the nucleus and activates the transcription of specific targets such as collagen, fibronectin, and $\alpha$-SMA. Alternatively, binding of TGF- $\beta$ with TGFR can also activate a wide variety of Smad-independent pathways (noncanonical signaling) to modify cell function $[70,71]$. TGF- $\beta$ induces renal fibrosis through several ways, including direct acting on fibroblasts and other cell types to induce ECM synthesis and reduce ECM degradation, and inducing the transition of various cell types into fibroblast-type cells that are capable of depositing ECM in injured kidneys. Under the condition of UUO injury, the protein level of TGF- $\beta$ in sample from kidney tissues was much higher than that in control sample, as determined by ELISA [36]. Moreover, the mRNA expression of TGF- $\beta$ in UUO kidney was also upregulated as determined by real-time RT-PCR [72]. Although the cell type, in which TGF- $\beta$ expression is activated, is unclear, these studies undoubtly showed the association of profibrotic TGF- $\beta$ with renal fibrosis. To inhibit the activation of TGF- $\beta$ signaling, various kinds of HDACi were applied in UUO-induced renal fibrosis. For example, Manson et al. [73] found that TSA inhibited the expression of TGF$\beta$-dependent profibrotic genes in a manner that depended on BMP receptor signaling.

Besides UUO model, activation of TGF- $\beta$ signaling was found in other animal models. In a hypertensioninduced kidney fibrosis model, Ang II induced the gene expression of TGF- $\beta$ in the mouse kidney both at transcriptional and translational level. However, this induction was blunt by a specific HDAC6 inhibitor or Smad3 knockdown, which suggested the profibrotic TGF- $\beta$ signaling was Smad-dependent [74]. Also, in a cell model of human renal mesangial cells treated aggregated IgA1 from IgAN patients, the protein levels of fibrosis markers and $\mathrm{pSmad} 2 / 3$ were increased, while pretreatment with the HDACi TSA or VPA partially reversed biological effects of aggregated IgA1 [46]. All these studies suggested activation of TGF- $\beta /$ Smad signal pathway contributes to renal fibrosis, while treatment of HDACi can reverse the signal pathway.

\section{Inhibition of EGRF Signaling and STAT3 Pathway}

The epidermal growth factor receptor (EGFR) is a transmembrane glycoprotein that is a member of the protein kinase superfamily. Binding of the protein to a ligand induces receptor dimerization and tyrosine autophosphorylation. Besides EGF, other ligands such as heparinbinding EGF-like growth factor and TGF- $\alpha$ can activate EGFR, which leads to the activation of ERK and STAT3, and subsequently the induction of various cellular re- 
sponses. Activation of EGRF signaling is involved in renal fibrosis. Either genetic or pharmacologic inhibition of EGFR significantly suppresses renal fibrosis in several animal models including Waved-2 mouse, UUO, Ang IIinduced renal fibrosis, and hyperuricemic nephropathy [75-77]. Moreover, activation of the EGFR can induce Smad3 phosphorylation and fibrotic response via reactive oxygen species-dependent mechanisms [78].

Some HDACis were found to inhibit EGRF signaling. By using selective inhibitor of SIRT-1 and/or -2, renal fibrosis was significantly attenuated in obstructive nephropathy, which was accompanied by dephosphorylation of EGFR and STAT3 [48]. Similarly, MS-275, a selective class I HDACi, also has shown to ameliorate renal fibrosis in UUO mice by suppressing phosphorylation and expression of EGFR and its downstream signaling molecule STAT3 [36]. Suberoylanilide hydroxamic acid, a Class I/II HDACi approved by US FDA for CTCL, was also found to reduce EGFR protein and mRNA in cultured proximal tubule cells and in diabetic kidneys [79]. Moreover, activation of EGFR and STAT3 was required for the proliferation of renal proximal tubular cells, while blocking of class I HDAC activity with a highly selective inhibitor or small interfering RNA resulted in reduced proliferation and suppressed EGFR expression and phosphorylation [23]. In addition, inhibition of HDAC6 with TSA could downregulate the expression of EGFR in Pkd1 mutant renal epithelial cells by an accelerated trafficking of EGFR [80]. These studies demonstrate the role of EGRF signaling in renal fibrosis and its inhibition by HDACi is a key molecular mechanism.

\section{Inhibition of JNK/Notch2 Signaling}

Notch signaling has an important role in kidney development and is downregulated in the adult kidney [81]. However, this signal pathway can be reactivated after renal injury. Expression of Notch pathway proteins was found to be correlated with the severity of fibrosis and the decline of renal function [82]. Moreover, expression of Notch in renal tubular cells was found to be both necessary and sufficient for fibrosis development and genetic deletion of the Notch pathway reduced renal fibrosis in a mouse model of folic acid-induced nephropathy [83]. More recently, Tung et al. [84] reported that TGF- $\beta 1$ stimulated expression of $\alpha$-SMA, fibronectin, phosphoJNK, and cleaved Notch-2 was significantly decreased by TSA treatment, while the levels of phospho-Smad2/3, phospho-p38, and phospho-ERK remained unchanged. They further demonstrated that administration of TSA or a $\gamma$-secretase inhibitor significantly ameliorated renal fi- brosis through suppression of the JNK/Notch-2 signaling activation in UUO mice. This work provided evidence that HDACi has the ability to inhibit JNK/Notch signaling and the crosstalk among different signaling pathways is involved in renal fibrosis.

\section{Conclusions}

Increasing evidence has clearly indicated that aberrant expressions of HDACs and subsequent epigenetic modifications play a crucial role in the pathogenesis of renal fibrosis. Therefore, the inhibition of HDAC enzymes with specific inhibitors might become an important therapeutic approach for chronic fibrotic kidney diseases. The TGF- $\beta$ signaling pathway plays a central role in renal fibrosis, and numerous HDACis prevent renal fibrosis by directly inhibiting this pathway. With the progress made in discovering noval HDACi, more potential HDACi will be applied in antifibrotic researches. As clinical trials with HDACis have shown promising results in antifibrotic therapy, it is rational to anticipate that less toxic isoformselective HDACis will improve clinical outcomes in the future.

\section{Acknowledgment}

We thank Dr. Ke Yang (Department of Nephrology, Xinqiao Hospital, Army Medical University) for critical reading of this manuscript.

\section{Disclosure Statement}

The authors declare that they have no potential conflicts of interest.

\section{Funding Sources}

This work was supported by research grants from the National Natural Science Foundation of China (Nos. 81400747 and 81700379).

\section{Author Contributions}

J.Z. and L.N. conceived the idea. L.N. and Y.L. analyzed the data. J.Z., L.N., and B.Z. wrote the manuscript. All authors approved the final manuscript. 


\section{References}

1 Liu BC, Tang TT, Lv LL, Lan HY. Renal tubule injury: a driving force toward chronic kidney disease. Kidney Int. 2018 Mar;93(3):568-79.

2 Lv W, Booz GW, Fan F, Wang Y, Roman RJ. Oxidative Stress and Renal Fibrosis: Recent Insights for the Development of Novel Therapeutic Strategies. Front Physiol. 2018 Feb 16; 9:105.

3 Nastase MV, Zeng-Brouwers J, Wygrecka M, Schaefer L. Targeting renal fibrosis: mechanisms and drug delivery systems. Adv Drug Deliv Rev. 2018 Apr;129:295-307.

4 Yang YM, Seki E. TNFa in liver fibrosis. Curr Pathobiol Rep. 2015 Dec;3(4):253-61.

5 Klinkhammer BM, Floege J, Boor P. PDGF in organ fibrosis. Mol Aspects Med. 2018 Aug; 62:44-62.

6 Strutz F, Zeisberg M, Hemmerlein B, Sattler B, Hummel K, Becker V, et al. Basic fibroblast growth factor expression is increased in human renal fibrogenesis and may mediate autocrine fibroblast proliferation. Kidney Int. 2000 Apr;57(4):1521-38.

7 Toda N, Mukoyama M, Yanagita M, Yokoi H. CTGF in kidney fibrosis and glomerulonephritis. Inflamm Regen. 2018 Aug;38(1):14.

8 Meng XM, Nikolic-Paterson DJ, Lan HY. TGF- $\beta$ : the master regulator of fibrosis. Nat Rev Nephrol. 2016 Jun;12(6):325-38.

9 Tang J, Liu N, Zhuang S. Role of epidermal growth factor receptor in acute and chronic kidney injury. Kidney Int. 2013 May;83(5): 804-10.

10 Buchtler S, Grill A, Hofmarksrichter S, Stöckert P, Schiechl-Brachner G, Rodriguez Gomez $\mathrm{M}$, et al. Cellular Origin and Functional Relevance of Collagen I Production in the Kidney. J Am Soc Nephrol. 2018 Jul;29(7):1859-73.

11 Larkin BP, Glastras SJ, Chen H, Pollock CA, Saad S. DNA methylation and the potential role of demethylating agents in prevention of progressive chronic kidney disease. FASEB J. 2018 Oct;32(10):5215-26.

12 Tampe B, Zeisberg M. Contribution of genetics and epigenetics to progression of kidney fibrosis. Nephrol Dial Transplant. 2014 Sep; 29 Suppl 4:iv72-9.

$13 \mathrm{Li} \mathrm{X}, \mathrm{Li} \mathrm{C}$, Sun G. Histone Acetylation and Its Modifiers in the Pathogenesis of Diabetic Nephropathy. J Diabetes Res. 2016;2016: 4065382.

14 Verdone L, Agricola E, Caserta M, Di Mauro E. Histone acetylation in gene regulation. Brief Funct Genomics Proteomics. 2006 Sep; 5(3):209-21.

15 Lyu X, Hu M, Peng J, Zhang X, Sanders YY. HDAC inhibitors as antifibrotic drugs in cardiac and pulmonary fibrosis. Ther Adv Chronic Dis. 2019 Jul;10:2040622319862697.

16 Schuetze KB, Stratton MS, Blakeslee WW, Wempe MF, Wagner FF, Holson EB, et al. Overlapping and Divergent Actions of Structurally Distinct Histone Deacetylase Inhibitors in Cardiac Fibroblasts. J Pharmacol Exp Ther. 2017 Apr;361(1):140-50.

17 Joanna F, van Grunsven LA, Mathieu V, Sarah S, Sarah D, Karin V, et al. Histone deacety- lase inhibition and the regulation of cell growth with particular reference to liver pathobiology. J Cell Mol Med. 2009 Sep;13(9b 9B):2990-3005

18 Mannaerts I, Nuytten NR, Rogiers V, Vanderkerken K, van Grunsven LA, Geerts A. Chronic administration of valproic acid inhibits activation of mouse hepatic stellate cells in vitro and in vivo. Hepatology. 2010 Feb; 51(2):603-14.

19 Choi HS, Song JH, Kim IJ, Joo SY, Eom GH, Kim I, et al. Histone deacetylase inhibitor, CG200745 attenuates renal fibrosis in obstructive kidney disease. Sci Rep. 2018 Aug; 8(1): 11546 .

20 Yang M, Chen G, Zhang X, Guo Y, Yu Y, Tian $\mathrm{L}$, et al. Inhibition of class I HDACs attenuates renal interstitial fibrosis in a murine model. Pharmacol Res. 2019 Apr;142:192-204.

21 Chun P. Therapeutic effects of histone deacetylase inhibitors on kidney disease. Arch Pharm Res. 2018 Feb;41(2):162-83.

22 Morigi M, Perico L, Benigni A. Sirtuins in Renal Health and Disease. J Am Soc Nephrol. 2018 Jul;29(7):1799-809.

23 Tang J, Yan Y, Zhao TC, Bayliss G, Yan H, Zhuang S. Class I histone deacetylase activity is required for proliferation of renal epithelial cells. Am J Physiol Renal Physiol. 2013 Aug; 305(3):F244-54.

24 Liu N, Zhuang S. Treatment of chronic kidney diseases with histone deacetylase inhibitors. Front Physiol. 2015 Apr;6:121.

25 Ponnusamy M, Zhuang MA, Zhou X, Tolbert E, Bayliss G, Zhao TC, et al. Activation of Sirtuin-1 Promotes Renal Fibroblast Activation and Aggravates Renal Fibrogenesis. J Pharmacol Exp Ther. 2015 Aug;354(2):142-51.

26 Ren Y, Du C, Shi Y, Wei J, Wu H, Cui H. The Sirt1 activator, SRT1720, attenuates renal fibrosis by inhibiting CTGF and oxidative stress. Int J Mol Med. 2017 May;39(5):1317-24.

27 Xue M, Li Y, Hu F, Jia YJ, Zheng ZJ, Wang L, et al. High glucose up-regulates microRNA-34a-5p to aggravate fibrosis by targeting SIRT1 in HK-2 cells. Biochem Biophys Res Commun. 2018 Mar;498(1):38-44.

28 He FF, You RY, Ye C, Lei CT, Tang H, Su H, et al. Inhibition of SIRT2 Alleviates Fibroblast Activation and Renal Tubulointerstitial Fibrosis via MDM2. Cell Physiol Biochem. 2018;46(2):451-60.

29 Li J, Qu X, Ricardo SD, Bertram JF, NikolicPaterson DJ. Resveratrol inhibits renal fibrosis in the obstructed kidney: potential role in deacetylation of Smad3. Am J Pathol. 2010 Sep;177(3):1065-71.

30 Kim JI, Jung KJ, Jang HS, Park KM. Genderspecific role of HDAC11 in kidney ischemiaand reperfusion-induced PAI-1 expression and injury. Am J Physiol Renal Physiol. 2013 Jul;305(1):F61-70.

31 Dong W, Jia Y, Liu X, Zhang H, Li T, Huang $\mathrm{W}$, et al. Sodium butyrate activates NRF2 to ameliorate diabetic nephropathy possibly via inhibition of HDAC. J Endocrinol. 2017 Jan; 232(1):71-83
32 Advani A, Huang Q, Thai K, Advani SL, White KE, Kelly DJ, et al. Long-term administration of the histone deacetylase inhibitor vorinostat attenuates renal injury in experimental diabetes through an endothelial nitric oxide synthase-dependent mechanism. Am J Pathol. 2011 May;178(5):2205-14.

33 Levine $\mathrm{MH}$, Wang Z, Bhatti TR, Wang Y, Aufhauser DD, McNeal S, et al. Class-specific histone/protein deacetylase inhibition protects against renal ischemia reperfusion injury and fibrosis formation. Am J Transplant. 2015 Apr;15(4):965-73.

34 Van Beneden K, Geers C, Pauwels M, Mannaerts I, Wissing KM, Van den Branden C, et al. Comparison of trichostatin $\mathrm{A}$ and valproic acid treatment regimens in a mouse model of kidney fibrosis. Toxicol Appl Pharmacol. 2013 Sep;271(2):276-84.

35 Marumo T, Hishikawa K, Yoshikawa M, Hirahashi J, Kawachi S, Fujita T. Histone deacetylase modulates the proinflammatory and -fibrotic changes in tubulointerstitial injury. Am J Physiol Renal Physiol. 2010 Jan; 298(1):F133-41.

36 Liu N, He S, Ma L, Ponnusamy M, Tang J, Tolbert E, et al. Blocking the class I histone deacetylase ameliorates renal fibrosis and inhibits renal fibroblast activation via modulating TGF-beta and EGFR signaling. PLoS One. 2013;8(1):e54001.

37 Tung CW, Hsu YC, Cai CJ, Shih YH, Wang CJ, Chang PJ, et al. Trichostatin A ameliorates renal tubulointerstitial fibrosis through modulation of the JNK-dependent Notch-2 signaling pathway. Sci Rep. 2017 Nov;7(1): 14495.

38 Noh H, Oh EY, Seo JY, Yu MR, Kim YO, Ha $\mathrm{H}$, et al. Histone deacetylase- 2 is a key regulator of diabetes- and transforming growth factor-betal-induced renal injury. Am J Physiol Renal Physiol. 2009 Sep;297(3):F729-39.

39 Lin W, Zhang Q, Liu L, Yin S, Liu Z, Cao W. Klotho restoration via acetylation of Peroxisome Proliferation-Activated Receptor $\gamma$ reduces the progression of chronic kidney disease. Kidney Int. 2017 Sep;92(3):669-79.

40 Choi SY, Ryu Y, Kee HJ, Cho SN, Kim GR, Cho JY, et al. Tubastatin A suppresses renal fibrosis via regulation of epigenetic histone modification and Smad3-dependent fibrotic genes. Vascul Pharmacol. 2015 Sep;72:13040.

41 He P, Li Z, Yue Z, Gao H, Feng G, Wang P, et al. SIRT3 prevents angiotensin II-induced renal tubular epithelial-mesenchymal transition by ameliorating oxidative stress and mitochondrial dysfunction. Mol Cell Endocrinol. 2018 Jan;460:1-13.

42 Ceccacci E, Minucci S. Inhibition of histone deacetylases in cancer therapy: lessons from leukaemia. Br J Cancer. 2016 Mar;114(6): 605-11.

43 Mishra N, Reilly CM, Brown DR, Ruiz P, Gilkeson GS. Histone deacetylase inhibitors modulate renal disease in the MRL-lpr/lpr mouse. J Clin Invest. 2003 Feb;111(4):539-52. 
44 Faria Freitas $\mathrm{M}$, Cuendet $\mathrm{M}$, Bertrand $\mathrm{P}$. HDAC inhibitors: a 2013-2017 patent survey. Expert Opin Ther Pat. 2018 Apr:1-17.

45 Chu QS, Nielsen TO, Alcindor T, Gupta A, Endo M, Goytain A, et al. A phase II study of SB939, a novel pan-histone deacetylase inhibitor, in patients with translocation-associated recurrent/metastatic sarcomas-NCIC-CTG IND 200†. Ann Oncol. 2015 May;26(5):97381.

46 Dai Q, Liu J, Du YL, Hao X, Ying J, Tan Y, et al. Histone deacetylase inhibitors attenuate $\mathrm{P}$-aIgA1-induced cell proliferation and extracellular matrix synthesis in human renal mesangial cells in vitro. Acta Pharmacol Sin. 2016 Feb;37(2):228-34.

47 Nguyễn-Thanh T, Kim D, Lee S, Kim W, Park SK, Kang KP. Inhibition of histone deacetylase 1 ameliorates renal tubulointerstitial fibrosis via modulation of inflammation and extracellular matrix gene transcription in mice. Int J Mol Med. 2018 Jan;41(1):95-106.

48 Ponnusamy M, Zhou X, Yan Y, Tang J, Tolbert E, Zhao TC, et al. Blocking sirtuin 1 and 2 inhibits renal interstitial fibroblast activation and attenuates renal interstitial fibrosis in obstructive nephropathy. J Pharmacol Exp Ther. 2014 Aug;350(2):243-56.

49 Kleaveland KR, Moore BB, Kim KK. Paracrine functions of fibrocytes to promote lung fibrosis. Expert Rev Respir Med. 2014 Apr; 8(2):163-72.

50 Williams SM, Golden-Mason L, Ferguson BS, Schuetze KB, Cavasin MA, Demos-Davies K, et al. Class I HDACs regulate angiotensin IIdependent cardiac fibrosis via fibroblasts and circulating fibrocytes. J Mol Cell Cardiol. 2014 Feb;67:112-25.

51 Pang M, Kothapally J, Mao H, Tolbert E, Ponnusamy M, Chin YE, et al. Inhibition of histone deacetylase activity attenuates renal fibroblast activation and interstitial fibrosis in obstructive nephropathy. Am J Physiol Renal Physiol. 2009 Oct;297(4):F996-1005.

52 Strutz F, Okada H, Lo CW, Danoff T, Carone RL, Tomaszewski JE, et al. Identification and characterization of a fibroblast marker: FSP1. J Cell Biol. 1995 Jul;130(2):393-405.

$53 \mathrm{Ng} \mathrm{YY,} \mathrm{Huang} \mathrm{TP,} \mathrm{Yang} \mathrm{WC,} \mathrm{Chen} \mathrm{ZP,} \mathrm{Yang}$ $\mathrm{AH}, \mathrm{Mu} \mathrm{W}$, et al. Tubular epithelial-myofibroblast transdifferentiation in progressive tubulointerstitial fibrosis in 5/6 nephrectomized rats. Kidney Int. 1998 Sep;54(3):86476.

54 Yang J, Liu Y. Dissection of key events in tubular epithelial to myofibroblast transition and its implications in renal interstitial fibrosis. Am J Pathol. 2001 Oct;159(4):1465-75.

55 Lovisa S, Zeisberg M, Kalluri R. Partial Epithelial-to-Mesenchymal Transition and Other New Mechanisms of Kidney Fibrosis. Trends Endocrinol Metab. 2016 Oct;27(10): 681-95.

56 Liu H, Xiong J, He T, Xiao T, Li Y, Yu Y, et al. High Uric Acid-Induced Epithelial-Mesenchymal Transition of Renal Tubular Epithelial Cells via the TLR4/NF-kB Signaling Pathway. Am J Nephrol. 2017;46(4):333-42.
57 Choi SY, Kee HJ, Kurz T, Hansen FK, Ryu Y, Kim GR, et al. Class I HDACs specifically regulate E-cadherin expression in human renal epithelial cells. J Cell Mol Med. 2016 Dec; 20(12):2289-98.

58 Yoshikawa M, Hishikawa K, Marumo T, Fujita $\mathrm{T}$. Inhibition of histone deacetylase activity suppresses epithelial-to-mesenchymal transition induced by TGF-betal in human renal epithelial cells. J Am Soc Nephrol. 2007 Jan;18(1):58-65.

59 Kang SW, Lee SM, Kim JY, Kim SY, Kim YH, $\mathrm{Kim} \mathrm{TH}$, et al. Therapeutic activity of the histone deacetylase inhibitor SB939 on renal fibrosis. Int Immunopharmacol. 2017 Jan;42: 25-31.

60 Khan S, Jena G, Tikoo K. Sodium valproate ameliorates diabetes-induced fibrosis and renal damage by the inhibition of histone deacetylases in diabetic rat. Exp Mol Pathol. 2015 Apr;98(2):230-9.

61 Khan S, Jena G. Sodium butyrate, a HDAC inhibitor ameliorates eNOS, iNOS and TGF$\beta 1$-induced fibrogenesis, apoptosis and DNA damage in the kidney of juvenile diabetic rats. Food Chem Toxicol. 2014 Nov;73:127-39.

62 Yang J, Liu Y. Blockage of tubular epithelial to myofibroblast transition by hepatocyte growth factor prevents renal interstitial fibrosis. J Am Soc Nephrol. 2002 Jan;13(1):96-107.

63 Nelson PJ, Rees AJ, Griffin MD, Hughes J, Kurts C, Duffield J. The renal mononuclear phagocytic system. J Am Soc Nephrol. 2012 Feb;23(2):194-203.

64 Kinugasa F, Noto T, Matsuoka H, Urano Y, Sudo Y, Takakura S, et al. Prevention of renal interstitial fibrosis via histone deacetylase inhibition in rats with unilateral ureteral obstruction. Transpl Immunol. 2010 May;23(12):18-23.

65 Eigl BJ, North S, Winquist E, Finch D, Wood L, Sridhar SS, et al. A phase II study of the HDAC inhibitor SB939 in patients with castration resistant prostate cancer: NCIC clinical trials group study IND195. Invest New Drugs. 2015 Aug;33(4):969-76.

$66 \mathrm{Ma}$, Jin X, He L, Wang Y. CXCL16 regulates renal injury and fibrosis in experimental renal artery stenosis. Am J Physiol Heart Circ Physiol. 2016 Sep;311(3):H815-21.

67 Wu WP, Tsai YG, Lin TY, Wu MJ, Lin CY. The attenuation of renal fibrosis by histone deacetylase inhibitors is associated with the plasticity of FOXP3+IL-17+ T cells. BMC Nephrol. 2017 Jul;18(1):225.

68 Ranganathan P, Hamad R, Mohamed R, Jayakumar C, Muthusamy T, Ramesh G. Histone deacetylase-mediated silencing of AMWAP expression contributes to cisplatin nephrotoxicity. Kidney Int. 2016 Feb;89(2):317-26.

69 Györfi AH, Matei AE, Distler JH. Targeting TGF- $\beta$ signaling for the treatment of fibrosis. Matrix Biol. 2018 Aug;68-69:8-27.

70 Pervan CL. Smad-independent TGF- $\beta 2$ signaling pathways in human trabecular meshwork cells. Exp Eye Res. 2017 May;158:13745.
71 Zhou XL, Xu P, Chen HH, Zhao Y, Shen J, Jiang $C$, et al. Thalidomide Inhibits TGF- $\beta 1$ induced Epithelial to Mesenchymal Transition in Alveolar Epithelial Cells via Smad-Dependent and Smad-Independent Signaling Pathways. Sci Rep. 2017 Nov;7(1):14727.

72 Chen J, Li D. Telbivudine attenuates UUOinduced renal fibrosis via TGF- $\beta / S m a d$ and $\mathrm{NF}-\kappa \mathrm{B}$ signaling. Int Immunopharmacol. 2018 Feb;55:1-8.

73 Manson SR, Song JB, Hruska KA, Austin PF. HDAC dependent transcriptional repression of Bmp-7 potentiates TGF- $\beta$ mediated renal fibrosis in obstructive uropathy. J Urol. 2014 Jan;191(1):242-52.

74 Batchu SN, Brijmohan AS, Advani A. The therapeutic hope for HDAC6 inhibitors in malignancy and chronic disease. Clin Sci (Lond). 2016 Jun;130(12):987-1003.

75 Liu N, Guo JK, Pang M, Tolbert E, Ponnusamy M, Gong R, et al. Genetic or pharmacologic blockade of EGFR inhibits renal fibrosis. J Am Soc Nephrol. 2012 May;23(5):854-67.

76 Chen J, Chen JK, Nagai K, Plieth D, Tan M, Lee TC, et al. EGFR signaling promotes TGF $\beta$-dependent renal fibrosis. J Am Soc Nephrol. 2012 Feb;23(2):215-24.

77 Liu N, Wang L, Yang T, Xiong C, Xu L, Shi Y, et al. EGF Receptor Inhibition Alleviates Hyperuricemic Nephropathy. J Am Soc Nephrol. 2015 Nov;26(11):2716-29.

78 Samarakoon R, Dobberfuhl AD, Cooley C, Overstreet JM, Patel S, Goldschmeding R, et al. Induction of renal fibrotic genes by TGF- $\beta 1$ requires EGFR activation, p53 and reactive oxygen species. Cell Signal. 2013 Nov;25(11): 2198-209.

79 Gilbert RE, Huang Q, Thai K, Advani SL, Lee $\mathrm{K}$, Yuen DA, et al. Histone deacetylase inhibition attenuates diabetes-associated kidney growth: potential role for epigenetic modification of the epidermal growth factor receptor. Kidney Int. 2011 Jun;79(12):1312-21

80 Liu W, Fan LX, Zhou X, Sweeney WE Jr, Avner ED, Li X. HDAC6 regulates epidermal growth factor receptor (EGFR) endocytic trafficking and degradation in renal epithelial cells. PLoS One. 2012;7(11):e49418.

81 Sharma S, Sirin Y, Susztak K. The story of Notch and chronic kidney disease. Curr Opin Nephrol Hypertens. 2011 Jan;20(1):56-61.

82 Murea M, Park JK, Sharma S, Kato H, Gruenwald A, Niranjan T, et al. Expression of Notch pathway proteins correlates with albuminuria, glomerulosclerosis, and renal function. Kidney Int. 2010 Sep;78(5):514-22.

83 Bielesz B, Sirin Y, Si H, Niranjan T, Gruenwald A, Ahn S, et al. Epithelial Notch signaling regulates interstitial fibrosis development in the kidneys of mice and humans. J Clin Invest. 2010 Nov;120(11):4040-54.

84 Tung CW, Hsu YC, Cai CJ, Shih YH, Wang CJ, Chang PJ, et al. Trichostatin A ameliorates renal tubulointerstitial fibrosis through modulation of the JNK-dependent Notch-2 signaling pathway. Sci Rep. 2017 Nov 3;7(1): 14495. 